\title{
Avaliação do modelo M-ROAMI: um experimento para a análise do seu potencial de ensino, interatividade e ubiquidade
}

\author{
Núbia dos S. R. Santana dos Santos ${ }^{1,2}$, Leandro Krug Wives ${ }^{1}$, \\ José Valdeni de Lima ${ }^{1}$, Alexandre Hauber da Silva ${ }^{1,3}$ \\ ${ }^{1}$ PPGIE / CINTED e Instituto de Informática \\ Universidade Federal do Rio Grande do Sul (UFRGS) \\ Porto Alegre - RS - Brazil \\ ${ }^{2}$ Universidade Federal Fluminense (UFF) \\ Rio de Janeiro - RJ - Brazil \\ ${ }^{3}$ Mecom do Brasil \\ Porto Alegre - RS - Brazil \\ nubia@sti.uff.br, \{valdeni, wives\} @inf.ufrgs.br, xandihds@hotmail.com
}

\begin{abstract}
In the development of educational content can be used digital and non-digital resources, as well as a hybrid model in order to expand the learning opportunities. In this context, this article presents an experiment with a Multimodal Learning Object developed in the M-ROAMI model with printed content (static) integrated digital content (and dynamic). The results obtained with the experiment show that the model can provide different opportunities for interaction and learning, through the ubiquitous manner available from the mobility of the media used.
\end{abstract}

Resumo. Na elaboração de um conteúdo educacional pode-se utilizar recursos digitais e não digitais, assim como um modelo híbrido com o intuito de ampliar às possibilidades de aprendizagem. Nesse contexto, este artigo apresenta um experimento realizado com um Objeto de Aprendizagem Multimodal desenvolvido no modelo M-ROAMI, com conteúdo impresso (estático) integrado a conteúdo digital (e dinâmico). Os resultados obtidos com o experimento evidenciam que o modelo pode oferecer diferentes oportunidades de interatividade e aprendizagem, de forma ubíqua a partir da mobilidade dos meios utilizados.

\section{Introdução}

Diante da variedade de recursos digitais e não digitais, o desenvolvimento de objetos de aprendizagem pode ser planejado para integrar recursos num modelo híbrido, que favoreça a exploração de conteúdos de diferentes formas. Dispositivos móveis, Ambientes Virtuais de Aprendizagem (AVAs), mídia impressa, conteúdos digitalizados, enfim, cada mídia fornece condições de aprendizagem, limitações e potencialidades.

Além disso, pressupõe-se que os alunos fazem escolhas diferentes quanto ao meio utilizado para estudo, pois cada um tem suas preferências e ritmos de 
aprendizagem. Nesse contexto, o presente artigo aborda o desenvolvimento de Objetos de Aprendizagem Multimodais (OAM) a partir da integração de recursos digitais e impressos usando dispositivos móveis, mídia impressa, códigos 2D e AVAs. Compreende-se que o desenvolvimento de Objetos de Aprendizagem Multimodais (OAM) deve ser planejado de modo a aproveitar as funcionalidades dos dispositivos móveis para promover uma aprendizagem ubíqua, inclusive, com auxílio de outras mídias. A integração de dispositivos com a mídia impressa permite o desenvolvimento de formas interessantes de exploração dos OAM, proporcionando aos alunos um estudo ubíquo. Ou seja, o acesso aos conteúdos educacionais independe do local e do horário onde os alunos se encontram. Vale ressaltar que cada tecnologia oferece vantagens e desvantagens, o que demonstra a relevância da integração e do uso de estratégias que promovam condições de explorar as vantagens de cada uma delas. Dessa forma, entende-se que recursos educacionais elaborados considerando a integração de tecnologias, como os OAMs, favorecem o ensino a distância, bem como o presencial.

Considerando um Objeto de Aprendizagem (OA) como "qualquer entidade, digital ou não digital, que possa ser utilizada, reutilizada ou referenciada durante o aprendizado suportado por tecnologias" (IEEE, 2002), entende-se que eles possam ser elaborados para uso nos dispositivos móveis mencionados anteriormente. Como exemplo de integração, pode-se citar o modelo de integração da mídia impressa com a TV digital visando ao acesso a conteúdos educacionais proposto por Gomes (2009). Por outro lado, percebe-se que a mídia impressa apresenta algumas desvantagens em relação ao uso de conteúdos dinâmicos, tais como animações, filmes e conteúdos interativos, por ser estática. Apesar disso, ela pode ser utilizada como mecanismo de ligação entre conteúdos estáticos com os conteúdos dinâmicos e digitalizados. Para integrar os benefícios da mídia impressa em papel às demais mídias pode-se agregar códigos 2D (duas dimensões) a um OA impresso, permitindo que esse seja utilizado como meio de interação, diferente de quando utilizado sem tais códigos. Uma vez que os recursos em papel estejam representados por códigos $2 \mathrm{D}$, o usuário é capaz de acessar os recursos multimídia de um OAM, desde que possua um dispositivo móvel capaz de reproduzi-lo, o que pode favorecer a interatividade e o acesso ao conteúdo interativo em qualquer hora e lugar. Conforme abordam Moreno e Mayer (2007), os ambientes de aprendizagem multimodais são ambientes que usam dois modos diferentes de representar o conteúdo: verbal e não verbal. Nesse caso, um OA pode ser considerado OAM quando permitir com que o usuário explore todos os sentidos através de informações verbais e não verbais.

Diante das possibilidades de integração de tecnologias, este artigo apresenta um experimento realizado para analisar o desempenho de alunos que utilizaram o modelo M-ROAMI - Modelo para Representação de Objetos Multimodais Interativos (Santos et al. 2013), elaborado para criação de OAM que permitam a aplicação de diferentes estratégias de ensino de acordo com as tecnologias disponíveis no momento da aprendizagem. Dessa forma, as opções estariam disponíveis para o aluno utilizar segundo as suas necessidades ou preferências, ou para uso pelo professor de acordo com a metodologia a ser aplicada. Compreende-se que a aprendizagem pode ocorrer de diferentes formas e, para isso, é fundamental apresentar opções diferenciadas de conteúdo na tentativa de atender às necessidades e expectativas de cada aluno. 
V Congresso Brasileiro de Informática na Educação (CBIE 2016)

Anais do XXVII Simpósio Brasileiro de Informática na Educação (SBIE 2016)

Este artigo está estruturado da seguinte forma: a seção 2 apresenta alguns trabalhos relacionados, a seção 3 detalha o modelo M-ROAMI e dados do experimento realizado usando o modelo; por fim, a seção 4 apresenta as considerações finais.

\section{Trabalhos relacionados}

Os dispositivos móveis têm sido muito utilizados para favorecer e complementar a aprendizagem. Dentre as vantagens no uso de dispositivos móveis pode-se destacar a mobilidade, interatividade e ubiquidade. Em relação à criação de ferramentas e estratégias para favorecer a aprendizagem com o apoio de dispositivos móveis e, em outros casos, o uso de AVAs, esta seção apresenta alguns trabalhos que possuem relação com esse contexto.

Bertolini et al. (2013) apresentaram a ferramenta iLaboratory, desenvolvida para o dispositivo iPhone da Apple, que funciona como um simulador de laboratório para reprodução de alguns experimentos de química. Filho, Aquino e Rosa (2013) apresentaram o projeto SIGAA Mobile, um sistema para Android que informatiza os procedimentos da área acadêmica através de diferentes módulos. Silva, Nóbrega e Jacob (2011) desenvolveram o software Labirinto do Rato, voltado para o desenvolvimento do raciocínio lógico infantil, onde a estratégia é utilizar o software como ferramenta educacional para os alunos que apresentam dificuldades de concentração. Já Orlandi e Isotani (2012) apresentaram o desenvolvimento de um sistema de autoria e distribuição de conteúdo educacional interativo para dispositivos móveis que permite armazenar listas de exercícios e as avaliações dos alunos. Dessa forma, listas de exercícios podem ser resolvidas no dispositivo, fornecendo a correção instantânea de um exercício ao assinalar uma de suas alternativas. Silva e Rebouças (2011) apresentaram o projeto MyQuimica, um jogo para auxiliar no ensino-aprendizagem de nomenclaturas químicas. Neto e Fonseca (2013) apresentaram um jogo educativo para Android desenvolvido com objetivo de apresentar, de uma forma diferente, situações e problemas que a criança precisasse mobilizar conhecimentos desenvolvidos em sala de aula. Minto, Santos e Junior (2013) apresentaram um aplicativo desenvolvido para permitir a criação de disciplinas, disponibilização de comunicados e avaliação de alunos.

Em relação à integração com AVAs, Sarmento et al. (2013) apresentaram uma arquitetura de comunicação entre dispositivos moveis e um AVA - o Solar 2.0, denominada ext-MobilisTTS, e uma aplicação cliente (Solar Mobilis) que se utiliza de recursos do hardware do dispositivo e de serviços externos a aplicação para oferecer mais interação entre professores e alunos. Já Fernandes et al. (2012) apresentaram uma proposta de integração de dispositivos móveis com o Moodle para aplicação de questionários. O trabalho de Forment et al. (2008) também apresenta o desenvolvimento de uma ferramenta para integração do Moodle com dispositivos móveis, o Moodlbile, que permite o acesso off-line a informações, as quais são armazenadas e sincronizadas com o servidor quando o usuário realiza o acesso on-line.

Todos os trabalhos mencionados utilizam dispositivos móveis e alguns deles integram tais dispositivos com AVAs, mas nenhum procura integrar mídia impressa e digital, tornando os OAs ubíquos. Nesse contexto, este artigo destaca o modelo MROAMI (Santos et al. 2013), que, assim como os trabalhos anteriores, utiliza dispositivos móveis e AVAs, mas inclui também a mídia impressa e códigos 2D. A 
V Congresso Brasileiro de Informática na Educação (CBIE 2016)

Anais do XXVII Simpósio Brasileiro de Informática na Educação (SBIE 2016)

seção a seguir apresenta um experimento realizado em sala de aula com o modelo MROAMI.

\section{Experimento usando o modelo M-ROAMI}

O modelo M-ROAMI (Santos et. al., 2013) foi proposto para integrar diferentes meios de explorar o mesmo recurso de aprendizagem, nesse caso aplicado com OAs. Foram incluídos computadores, dispositivos móveis, a mídia impressa e códigos 2D. Pelo fato de a mídia impressa ainda ser muito utilizada para conteúdo educacional, tanto no ensino a distância como no ensino presencial, o modelo inclui a utilização de mídia impressa em virtude disso e das características relevantes dessa mídia na aprendizagem. Cabe salientar que Sellen e Harper (2001) ressaltam quatro características-chave do papel, como a possibilidade de navegação rápida e flexível através de um documento, a inserção de anotações durante a leitura, a mobilidade e a integração de atividades híbridas, tais como, escrita e leitura. O objetivo do modelo é oferecer a mobilidade e interatividade do conteúdo educacional, permitindo a continuidade das atividades independentemente do meio e local utilizados. Para integração de recursos com a mídia impressa foram utilizados os códigos 2D QR Code ${ }^{1}$, os quais permitem o carregamento de informações na direção vertical, bem como na horizontal. Dessa forma, os OAMs podem ser acessados pelo dispositivo móvel, pelo computador e pela mídia impressa, através de sua materialização, isto é, o OA impresso com os recursos dinâmicos (vídeo, áudio, animação, atividades, etc.) representados pelos códigos 2D. Além disso, foram desenvolvidas aplicações para suporte ao modelo no que tange a materialização automática em mídia impressa, registro de informações (representadas por QR Codes) nos dispositivos móveis de forma off-line e atualização de respostas (registros) no AVA Moodle (quando on-line), conforme apresenta a arquitetura (Figura 1).

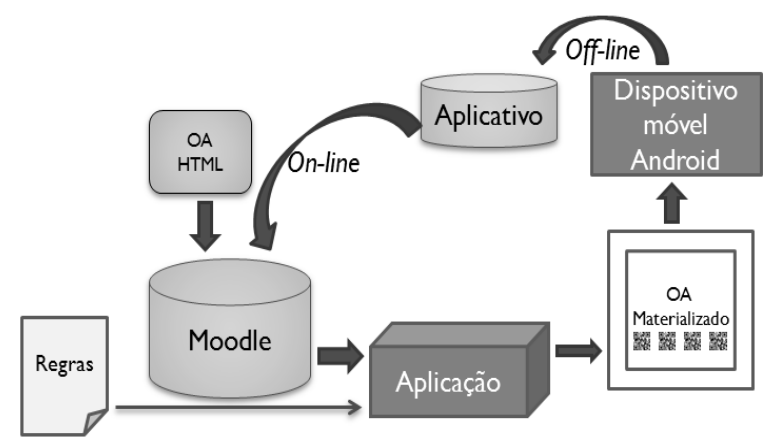

Figura 1. Arquitetura do modelo proposto

As aplicações desenvolvidas foram implementadas para recursos do tipo questionário como teste inicial do modelo proposto. No Moodle, um questionário pode conter questões de múltipla escolha, verdadeiro/falso, discursiva, entre outras. Para o teste com a materialização automática de questionários foi desenvolvida uma aplicação em PHP para gerar o conteúdo materializado com QR Codes. A aplicação exibe todos os questionários criados pelo usuário, com a opção de gerar um documento em formato PDF, com a materialização do questionário, o qual poderá ser utilizado com o aplicativo Android. Dentre algumas funções do aplicativo desenvolvido pode-se destacar a decodificação do recurso representado pelo código $2 \mathrm{D}$, nesse caso questionários, o

\footnotetext{
${ }^{1}$ QR Code é marca registrada da DENSO WAVE INCORPORATED.
} 
V Congresso Brasileiro de Informática na Educação (CBIE 2016)

Anais do XXVII Simpósio Brasileiro de Informática na Educação (SBIE 2016)

registro das respostas, o feedback off-line e o envio/atualização das respostas no Moodle. Para o desenvolvimento do aplicativo foi utilizada a linguagem Java. A comunicação dos dados foi realizada através da tecnologia JSON (JavaScript Object Notation), para fazer a validação do usuário, ou seja, utilizar as informações de login no Android e validar com o banco de dados do Moodle, para envio das informações do questionário e das questões respondidas.

\subsection{Análise de desempenho dos alunos}

O modelo foi aplicado em uma turma de graduação em Computação da Universidade Federal do Rio Grande do Sul, na disciplina Classificação e Pesquisa de Dados, modalidade presencial. Os alunos responderam a um questionário inicial sobre $\mathrm{o}$ conteúdo de Árvores B, as quais são árvores balanceadas de pesquisa projetadas para minimizar o tempo de acesso em buscas, inserções e remoções de registros em grandes arquivos de dados. Para apresentar o conteúdo da disciplina, foi desenvolvido em HTML 5, seguindo o modelo M-ROAMI, o OAM 'Árvores'. Esse foi posteriormente materializado em mídia impressa com códigos 2D representando os recursos dinâmicos, i.e., vídeos, animações, dicas, etc. Essa materialização é feita automaticamente com o apoio de softwares desenvolvidos dentro do contexto do M-ROAMI. Dessa forma, o OA poderia ser utilizado diretamente no computador, no dispositivo móvel e, ainda, na mídia impressa com suporte de dispositivos móveis para decodificar os códigos $2 \mathrm{D}$. Na segunda aula, a turma foi dividida em dois grupos, onde o Grupo 1 estudou o conteúdo do OAM na mídia impressa e o Grupo 2 estudou o OAM materializado, com códigos 2D, e dispositivos móveis. Dessa forma, no planejamento do experimento foram consideradas as atividades:

- Aula 1: todos os alunos responderam o questionário inicial sobre Árvores B;

- Aula 2: a turma foi dividida em dois grupos: o Grupo 1 explorou o OAM na mídia impressa, realizou as atividades do OAM e respondeu o questionário impresso sobre o conteúdo; o Grupo 2 explorou o OAM materializado usando a mídia impressa e o dispositivo móvel, realizou as atividades do OAM, respondeu o questionário sobre o conteúdo utilizando o aplicativo Android, enviando as respostas automaticamente para o Moodle.

A Figura 2 apresenta o OAM Árvores. A interface do OAM foi elaborada considerando o modelo de metadados UMBRELO (Santos et al., 2012), o qual foi estendido para adequação de alguns recursos ao modelo. 
V Congresso Brasileiro de Informática na Educação (CBIE 2016)

Anais do XXVII Simpósio Brasileiro de Informática na Educação (SBIE 2016)

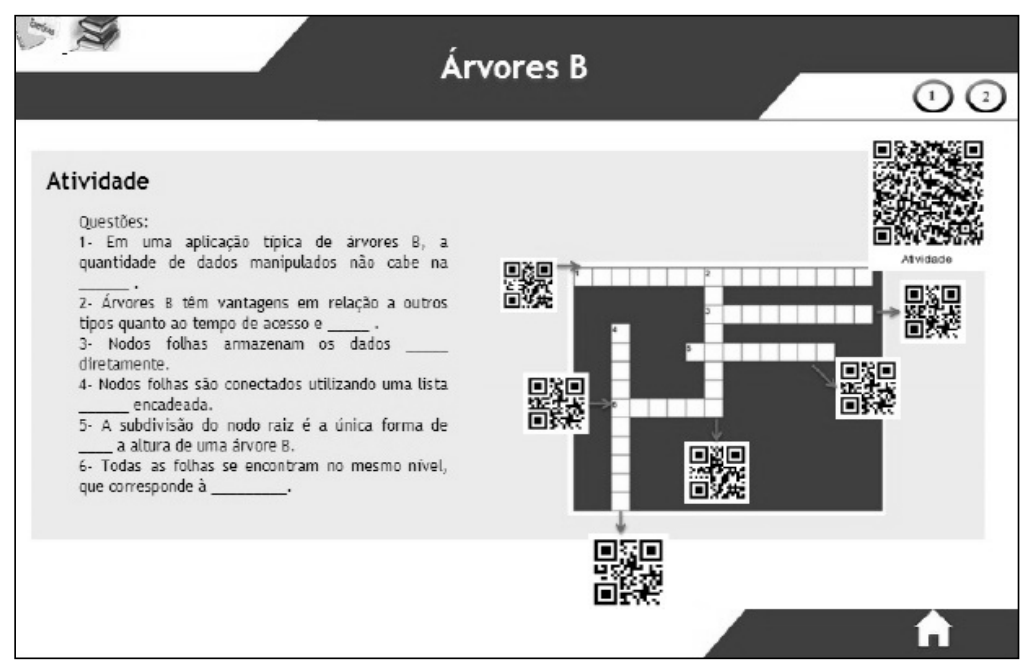

Figura 2. OAM Árvores materializado com QR Codes

Após a utilização do OAM em sala de aula os dois grupos responderam outro questionário sobre Árvores B. Dessa forma, pôde-se verificar a diferença de desempenho dos alunos antes e após a utilização do OAM. Com os resultados dos questionários (15 questões) respondidos pelos alunos, pôde-se comparar o desempenho de cada grupo para verificar se a diferença antes e depois da utilização do OAM foi significativa e em seguida comparar os dois grupos. Nos resultados obtidos, observou-se que a média de acertos do Grupo 1 no questionário 2 foi superior à média de acertos do mesmo grupo no questionário 1 (Figura 3).

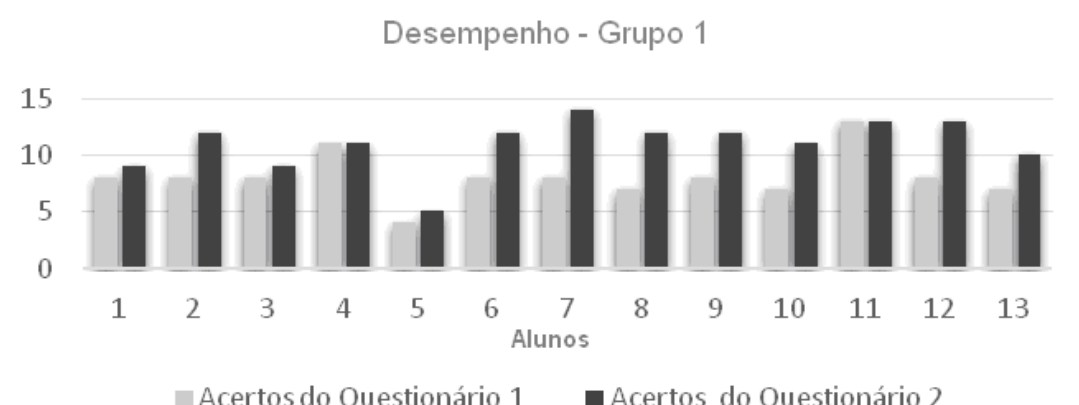

Figura 3. Resultado do questionário (antes e depois do OAM) do Grupo 1

A média do Questionário 1 respondido pelo Grupo 1 foi de 8,1 acertos (num total de 15) enquanto a média do mesmo grupo no Questionário 2 foi 11 . O teste de hipótese com $t$-student para amostras emparelhadas foi aplicado para verificar se a diferença foi significativa. Vale ressaltar que, no Grupo 1, os alunos que responderam o Questionário 1 foram os mesmos que responderam o Questionário 2. O mesmo procedimento foi definido para o Grupo 2. Nesse caso, são amostras dependentes (emparelhadas). Então, o teste verificou se a média da diferença de desempenho foi significativamente maior do que zero. Ou seja: $\mathrm{H}_{0}: \mu_{\mathrm{d}}=0$ e $\mathrm{H}_{1}: \mu_{\mathrm{d}}<0$. Aplicando o $t$ student foi verificado que o $t$ crítico $(-1,78)$ foi maior do que o $t$ observado $(-5,11)$. Considerando a uni-caudal à esquerda, então, rejeitou-se a hipótese que a média das diferenças fosse 0 , ou seja $-t<-t$ crítico - rejeita-se $\mathrm{H}_{0}$. $\mathrm{O}$ mesmo procedimento foi 
V Congresso Brasileiro de Informática na Educação (CBIE 2016)

Anais do XXVII Simpósio Brasileiro de Informática na Educação (SBIE 2016)

realizado com o Grupo 2 com o objetivo de verificar se houve diferença de desempenho antes e depois da utilização do OAM (Figura 4)

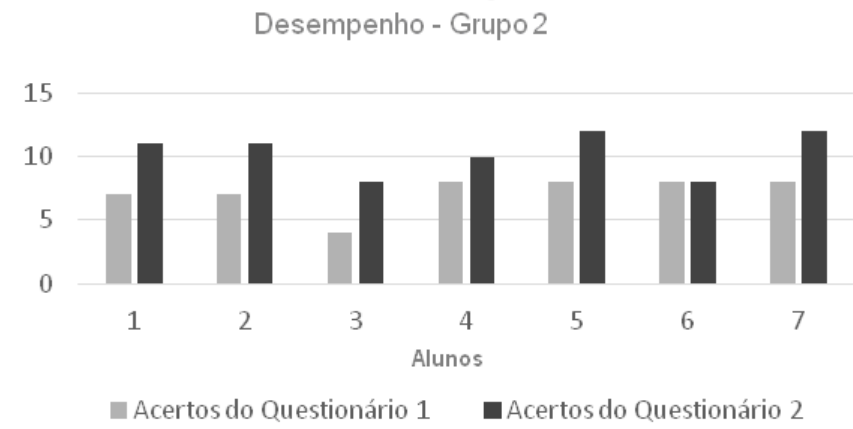

Figura 4. Resultado do questionário (antes e depois do OAM) do Grupo 2

A média do Questionário 1 respondido pelo grupo 2 foi de 7,14 (num total de 15 questões), enquanto a média do mesmo grupo no Questionário 2 foi 10,28. Aplicando o $t$-student no Grupo 2, foi verificado que o $t$ crítico $(-1,94)$ foi maior do que o $t$ observado $(-5,28)$. Dessa forma, considerando a uni-caudal à esquerda, rejeitou-se a hipótese que a média das diferenças fosse 0 , ou seja $-t<-t$ crítico - rejeita-se $\mathrm{H}_{0}$. Com esse resultado, verifica-se que a diferença de ambos grupos em relação ao primeiro e segundo questionário foi significativa.

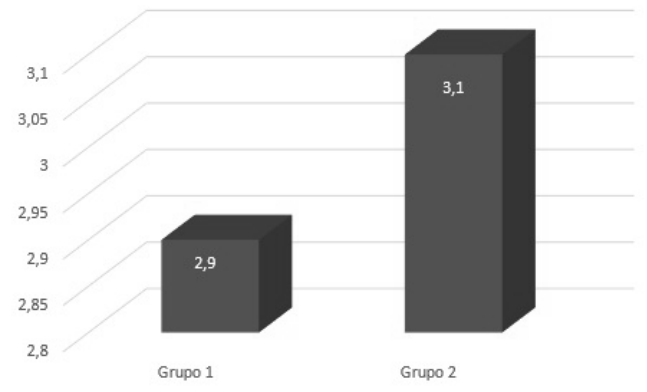

Figura 5. Diferença entre os questionários em cada grupo

Analisando a média da diferença entre os questionários dos dois grupos, verificou-se que o Grupo 2 obteve uma média de diferença maior do que o Grupo 1. Esse resultado pode indicar um melhor desempenho do Grupo 2, após o uso do OAM.

\subsection{Análise da interatividade do modelo proposto}

Após a análise de desempenho foi solicitado aos alunos que respondessem a um questionário sobre o método e os recursos utilizados na aula. $\mathrm{O}$ Grupo 1 respondeu o questionário de interatividade do OAM considerando os recursos utilizados, no caso, a mídia impressa. O mesmo foi solicitado ao Grupo 2 que utilizou o OAM materializado com dispositivos móveis. Para isso, foi elaborado um questionário com vinte e cinco itens referentes à interatividade e princípios da Aprendizagem Multimídia (Mayer, 2005), tais como princípio da multimídia, princípio da proximidade espacial, princípio da contiguidade temporal, princípio da coerência, princípio da sinalização, princípio da modalidade, princípio da redundância e personalização. Também foram analisados os tipos de interatividade apresentados por Moreno e Mayer (2007), sendo eles: dialogar, 
V Congresso Brasileiro de Informática na Educação (CBIE 2016)

Anais do XXVII Simpósio Brasileiro de Informática na Educação (SBIE 2016)

controlar, manipular, pesquisar e navegar. No questionário de interatividade foi utilizada a escala de Likert com as opções: Sim (S), Parcialmente com Poucas restrições $(\mathrm{P}+)$, Parcialmente $(\mathrm{P})$, Parcialmente com Muitas restrições (P-) e Não (N).

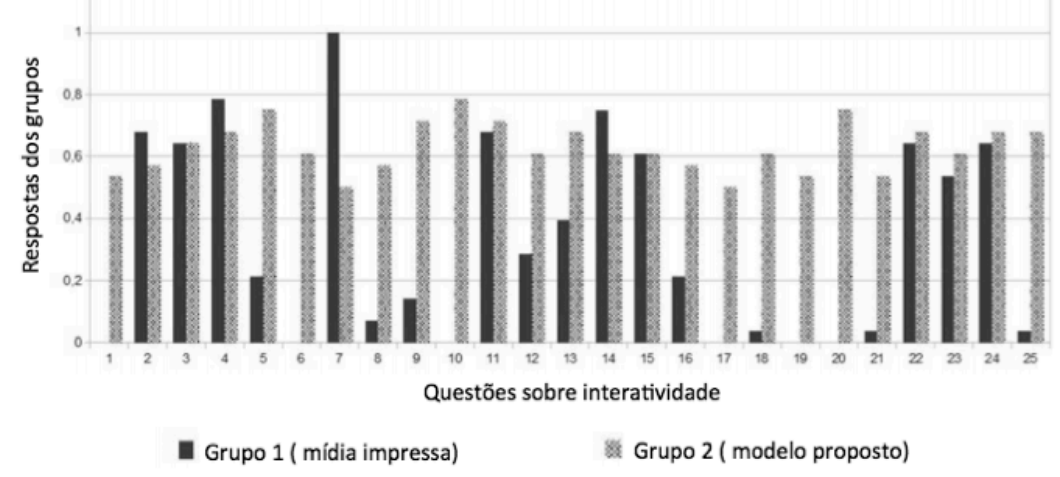

Figura 6. Respostas dos grupos sobre a interatividade do OAM

Os resultados obtidos com a aplicação do questionário sobre a interatividade (Figura 6) evidenciaram que:

- O Grupo 2 apresentou respostas mais positivas que o Grupo 1, o que pode indicar mais interatividade do material ao usar o modelo proposto, i.e., integração das tecnologias: dispositivos móveis, mídia impressa e códigos 2D;

- No item 7 sobre "O meio utilizado para acesso ao OA permite realizar anotações", o Grupo 1 apresentou mais respostas positivas que o Grupo 2. Vale ressaltar que o OAM materializado também integra vantagens da mídia impressa tais como facilidade de leitura e anotações;

- Outros itens em destaque para a mídia impressa, de acordo com o Grupo 1, foram os itens 2, 4 e 14 que representam a contribuição para o entendimento do conteúdo abordado, a realização de atividades e a facilidade de manipulação do conteúdo, respectivamente;

- Nos demais itens, o Grupo 2 apresentou respostas mais satisfatórias em relação à interatividade do que o Grupo 1, que utilizou apenas a mídia impressa.

Dessa forma, pôde-se verificar que o Grupo 2 apresentou mais concordância em relação às questões afirmativas (positivas) sobre a interatividade e relevância do OAM. Em alguns itens do questionário verifica-se valores correspondentes a zero ("não") informados pelo Grupo 1. Nesse caso, vale destacar que o OAM usado somente na mídia impressa não proporciona o acesso aos mesmos recursos que um OAM materializado com códigos 2D com suporte de um dispositivo móvel. A aplicação do questionário colaborou para evidenciar a diferença em relação à percepção de interatividade dos grupos, apresentando resultados que podem indicar que o OAM materializado com códigos 2D pode oferecer mais condições de aprendizagem e interatividade do que a mídia impressa utilizada separadamente.

\section{Considerações finais}

A partir deste experimento foi evidenciado que o uso de códigos $2 \mathrm{D}$, tal como o $\mathrm{QR}$ Code, proporciona mais interatividade ao conteúdo impresso, quando o aluno utiliza o 
dispositivo móvel como meio complementar. Dessa forma, o uso integrado desses recursos, conforme propõe o modelo M-ROAMI, possibilita que o aluno utilize o material digital ou impresso para estudo e tenha acesso ao conteúdo dinâmico, a qualquer hora e em qualquer lugar. Compreende-se que os recursos dinâmicos tais como vídeo, animação, áudio, complementares e representados por QR Code no material impresso, podem facilitar o entendimento sobre o assunto tornando o estudo mais vasto e agradável. A estratégia utilizada para realização do experimento permitiu verificar o desempenho dos alunos, de grupos distintos, antes e após a utilização do OAM. Independentemente do meio utilizado para estudo, o resultado apresentou melhor desempenho dos alunos após a utilização do OAM. No entanto, o grupo que utilizou o OAM materializado - na mídia impressa - com dispositivos móveis apresentou uma média superior de desempenho. A diferença entre os resultados dos dois grupos pode indicar a relevância da integração de tecnologias ao permitir a facilidade de acesso às outras formas de informação e recursos complementares. Vale ressaltar a necessidade de uma análise do contexto, do perfil de alunos e preferência, da complexidade do conteúdo, enfim, de todas as variáveis que direcionam para a seleção de uma mídia para disponibilização de conteúdos educacionais. Por isso, entende-se que na elaboração de um OAM deve-se considerar tais variáveis, de modo a favorecer o uso ubíquo em diferentes situações, estratégias e ambientes de aprendizagem.

O resultado também indica, a partir da percepção dos alunos, aspectos positivos de cada mídia, o que enseja a integração de recursos como forma de expandir as possibilidades de ensino e aprendizagem. Na realização da aula algumas dificuldades foram encontradas, tais como o número reduzido de alunos pertencentes ao Grupo 2 devido à necessidade de recursos específicos para uso do dispositivo móvel, i.e., compatibilidade com o leitor de QR Code e aplicativo Android, além do acesso à Internet. Como trabalhos futuros, pode-se realizar estudos que contemplem um comparativo dos alunos em outras disciplinas para fins de validação das hipóteses, assim como uma análise qualitativa, usando um OAM e o modelo M-ROAMI, de preferência com a evolução do aplicativo Android, adicionando novas funcionalidades, bem como da aplicação para materialização de todos os recursos dinâmicos de OAM através de códigos $2 \mathrm{D}$.

\section{Referências}

Bertolini et al (2013), Laboratório Virtual Interativo para reprodução de Experimentos de Química através de Dispositivos Móveis, XXIV Simpósio Brasileiro de Informática na Educação- SBIE, Campinas.

Fernandes, K. T. et al, (2012) Question Mobile: Ampliando Estratégias de Avaliação da Aprendizagem por Meio de Dispositivos Móveis, Anais do XXIII SBIE - Simpósio Brasileiro de Informática na Educação, Rio de Janeiro.

Filho, I.B., Aquino, G., Rosa, J.G.S. (2013), SIGAA Mobile - O caso de sucesso da ferramenta de gestão acadêmica na era da computação móvel, XXIV Simpósio Brasileiro de Informática na Educação - SBIE, Campinas.

Forment, A.M.,Guerrero, M.J.G.(2008) Moodlbile: Extending Moodle to the Mobile On/Offline Scenario. In: Proceedings of IADIS International Conference Mobile Learning. Algarve, Portugal 
V Congresso Brasileiro de Informática na Educação (CBIE 2016)

Anais do XXVII Simpósio Brasileiro de Informática na Educação (SBIE 2016)

Gomes, F. J. L., (2009) Explorando Objetos de Aprendizagem na TV digital: estudo de caso de alternativas de interação, Estado do Rio Grande do Sul. Porto Alegre: PGIE/UFRGS, 2009 162p. Tese de Doutorado.

IEEE, L.T.S.C., (2002) IEEE Standard for learning object medadata. Disponível em:< http://ltsc.ieee.org/wg12/index.html> Acesso em: 28 Set. 2010

Mayer, R. E. (2005) Introduction to Multimedia Learning. In: MAYER, R. E. (Ed.). The Cambridge Handbook of Multimedia Learning. New York: Cambridge University Press.

Minto, R. S.,Santos, V. F. M., Paschoal Júnior, F. (2013) Aplicação M-Learning em Android, ICBL2013 - International Conference on Interactive Computer aided Blended Learning, CEFET-RJ/DEPIN, Rio de Janeiro.

Moreno, R., Mayer, R. (2007) Interactive Multimodal Learning Environments, Special Issue on Interactive Learning Environments: Contemporary Issues and Trends, Educ Psychol Rev.

Neto, J.F.B., Fonseca, F.S. (2013), Jogos educativos em dispositivos móveis como auxílio ao ensino da matemática, Renote- Novas Tecnologias na Educação, v.11 no 1.

Orlandi, B.H.; Isotani, S. (2012) Uma Ferramenta para Distribuição de Conteúdo Educacional Interativo em Dispositivos Móveis, Anais do XXIII SBIE - Simpósio Brasileiro de Informática na Educação, Rio de Janeiro.

Santos, N.S.R.S.; Lima, J.V.; Wives, L.K.; Silva, A. H. (2013) M-ROAMi - Um Modelo para Promover o Desenvolvimento de Objetos de Aprendizagem Multimodais, Anais do XXIV Simpósio Brasileiro de Informática na Educação SBIE, Campinas.

Santos, N.S.R.S et al (2012), Metadados para Objetos de Aprendizagem: prova de conceito do modelo UMBRELO, Anais do XXIII SBIE - Simpósio Brasileiro de Informática na Educação, Rio de Janeiro

Sarmento et al. (2013), extMobilisTTS: Uma Arquitetura de Aplicação Móvel para Suporte a Foruns usando Text-to-Speech em Ambientes Virtuais de Aprendizagem, 31 Simpósio Brasileiro de Redes de Computadores e Sistemas Distribuídos - SBRC, p. $1068-1075$

Sellen, A., Harper, R. (2001) The Myth of the Paperless Office.Cambridge, MA:MIT Press.

Silva, D.L.M, Rebouças, A.D.D.S. (2011) MyQuímica - Um jogo para auxiliar no ensino aprendizagem das nomenclaturas químicas, Anais do XXII SBIE-Simpósio Brasileiro de Informática na Educação, Aracaju.

Silva, S.S.R., Nobrega, S.M., Jacob Jr. A.F.L. (2011) Labirinto do Rato: jogo educacional infantil para dispositivos móveis, Anais do XXII SBIE - Simpósio Brasileiro de Informática na Educação, Aracaju. 